\title{
Emerging Optical Fibre Technologies with Potential Defence Applications
}

\author{
Wei H. Loh*, Dan Hewak, Marco N. Petrovich, John R Hayes, Will Stewart, Andy Clarkson \\ Optoelectronics Research Centre, University of Southampton, Highfield, Southampton, SO17 1BJ, \\ UK
}

\begin{abstract}
Recent years have seen the development of a range of promising optical fibre technologies emerge, enabled by advances in materials and fabrication techniques. We describe 3 emerging areas in optical fibre developments: nanomechanical optical fibres, microstructured hollow core silica fibres for high peak optical power and/or extended infrared transmission, and chalcogenide glasses and fibres for mid-IR applications.
\end{abstract}

Keywords: optical fibre, microstructured fibre, laser fibre delivery, chalcogenide fibre.

\section{INTRODUCTION}

Optical fibres have progressed and evolved quite considerably from the standard all-glass core-clad fibre structures used in optical telecommunications, both in design as well as materials. This progress is the result of a combination of technology push arising from advances made in fabrication techniques, and applications pull for servicing a broader range of fibre functionalities. We describe here some of the more recent developments, ranging from mechanically movable cores for reconfigurable networks and sensors, air-filled core fibres for reduced material optical nonlinearity and absorption loss, and infrared chalcogenide glass fibres for long wavelength transmission.

\section{NANOMECHANICAL OPTICAL FIBRES}

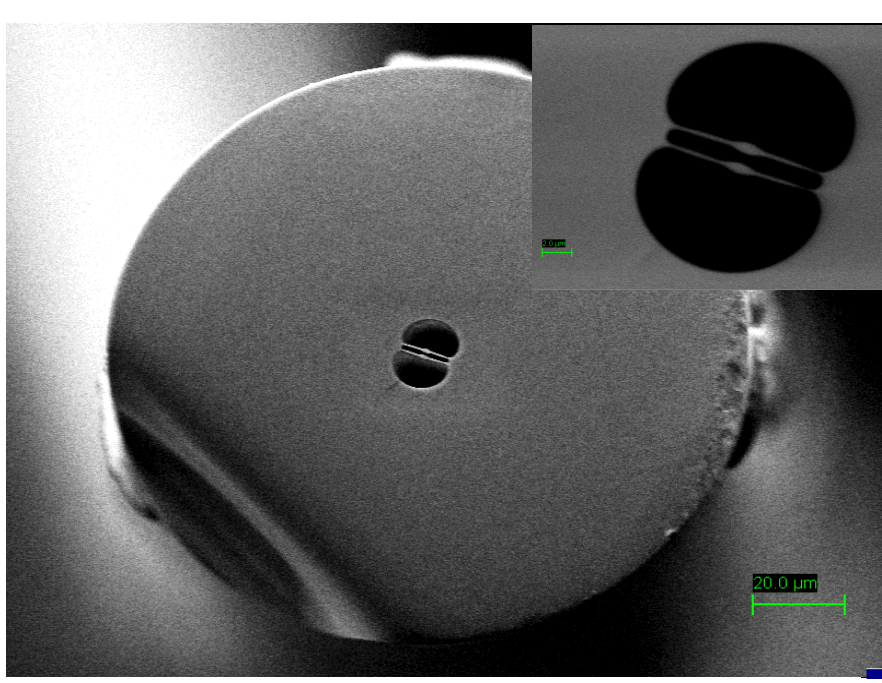

Figure 1. SEM photograph of a suspended dual core optical fibre with the elliptical cores shown in the inset suspended by two thin glass membranes

*Email address: whl@orc.soton.ac.uk
We first describe the successful realisation of nanomechanical optical fibres, i.e. fibres where the core does not only transmit light, but is engineered to be capable of controlled nanometre-scale mechanical movement [1]. We have fabricated fibres with two movable cores close enough to each other (Figure 1) to be optically coupled, thereby acting as a directional coupler. The sub-micron sized cores and core separation dimensions were chosen so that the two cores are optically coupled through their evanescent fields. Each core is held suspended in air from the outer glass cladding by two glass membranes which are $200 \mathrm{~nm}$ thick, such that the cores can move freely in the direction perpendicular to the membranes. The cores are elliptical because they become elongated during the fibre drawing, due to surface tension forces which are substantial at these small dimensions. 
The opto-mechanical functionality of the fibre is achieved through altering the geometry and consequently the optical coupling length, enabling optical switching of the light by moving one of the cores several nanometres. Such fibres can also be used to detect very small changes in the environment, e.g. pressure or vibration, which will appeal to the field of sensing. Sub-micron diameter optical fibres have previously been fabricated by direct drawing or tapering to yield standalone single core fibres. While attractive for their evanescent field properties, post-fabrication assembly is required for more complex multi-fibre configurations. What our work demonstrates here is that low-loss sub-micron optical fibres can be directly drawn on a draw tower with a conventional $125 \mu \mathrm{m}$ glass cladding for protection, while containing multiple fibre cores within the cladding that are capable of optical and mechanical interaction.

Mechanical actuation in suitably small structures is well-known in Silicon MEMS (Microelectromechanical systems) and NEMS (Nanoelectromechanical Systems) devices, and these have had wide impact in a range of applications, e.g. as accelerometers and gyroscopes, and for displays. The current work can also be viewed as a development towards a new fibre-based MEMS technology. With fibre-MEMS, we have the manufacturing advantage of optical fibres, where long device lengths can be fabricated by drawing from a fibre draw tower, with low optical transmission losses. With much longer device lengths than is practical on a silicon chip, the sensitivity of these fibre-based devices to motion or changes in the environment will be correspondingly greater. Our results show that such fibres are capable of responding optically to nanometre movements in the fibre cores.

\section{Fibre Functionality}
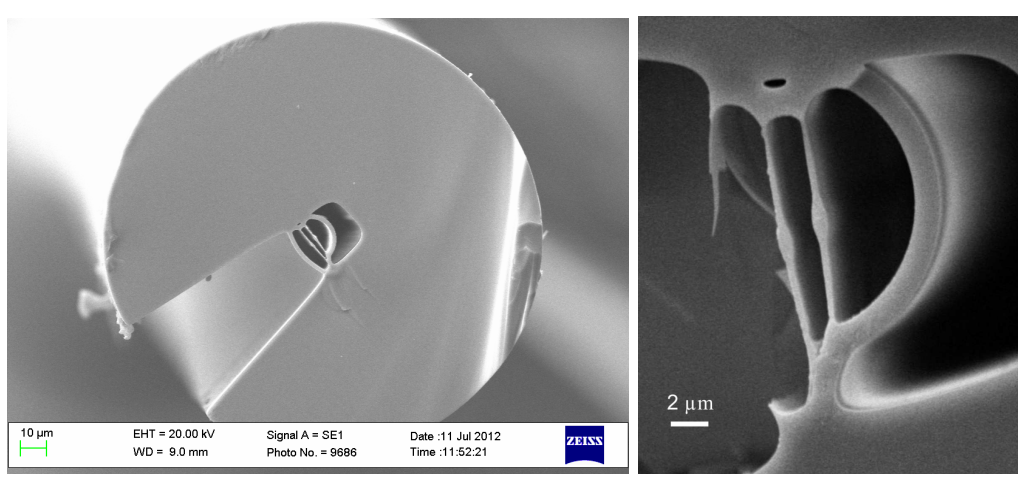

Figure 2. SEM photograph of a suspended dual core optical fibre with a channel fabricated to the outside of the fibre. Left: SEM photo of cross-section of the fibre after drawing from the fibre draw tower.

Right: Fibre cross-section showing core region after the separating wall has heen etched to nrovide access to the ontside
As an initial demonstration to exploit the functionality in these fibres, we fabricate an additional channel through the fibre cladding, to provide direct access to the environment from the open hole adjacent to one core, by etching through a portion of the cladding wall (Figure 2). Creating this access channel allows us to apply direct pressure selectively to one core, and demonstrate optical switching.

A $43 \mathrm{~cm}$ length of the fibre, with a $3 \mathrm{~cm}$ section in the middle of the fibre etched through to allow direct access to one core was placed in a pressure chamber. Light from a $1550 \mathrm{~nm}$ wavelength pigtailed laser diode is collimated, sent through a polarizer, and focused into one of the fibre cores. The polarization state of the light was parallel to the long elliptical axis of the core; this polarization state was selected as it has a lower optical propagation loss $(2.54 \mathrm{~dB} / \mathrm{m})$ than the orthogonally polarized state $(3.30 \mathrm{~dB} / \mathrm{m})$. The light output from the fibre was imaged onto an IR video camera, and the optical intensity pattern continuously monitored as the pressure applied to the fibre core was varied.
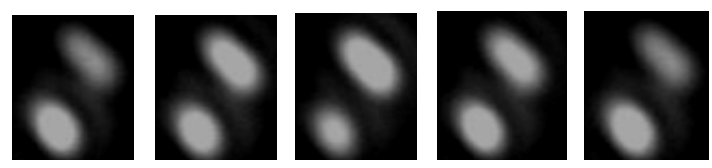

Figure 3 shows the change in the optical intensity pattern emitted from the dual core fibre as the pressure was increased by $100 \mathrm{mbar}$. It is clearly seen that the light intensity switches from one core to the other and back again. This same periodicity was also observed over the full pressure range of $250 \mathrm{mbar}$ afforded by our Figure 3. IR video camera images of the optical intensity from the output of the suspended dual core fibre as the pressure applied to one of the cores is increased bv experimental set-up. The change in optical pattern is readily repeatable, and showed the same behaviour for both increasing and decreasing pressure conditions.

Using the dimensions of the fibre structure from the SEM photos, the refractive index and bulk Young's modulus of the glass, and the applied pressure readings, we have modelled the optical behaviour, and calculated the amount of mechanical movement imparted to the core for the optical switching response seen in Figure 3. For the fibre dimensions 
of Figure 2, we have found that the core only needs to move by $8 \mathrm{~nm}$ for the light output to switch from one core to the other.

\section{MICROSTRUCTURED HOLLOW CORE FIBRES}

Microstructured optical fiber technology allows for low loss transmission in a hollow core, either through a bandgap effect (Photonic Bandgap Fibres) or through anti-resonant guidance (Kagome-type fibres). Photonic Bandgap Fibers (PBGFs) contain complex photonic crystal cladding structures composed of 5-7 rings of highly expanded, identical air holes, surrounding a hollow core that is formed by omitting a number of elements (typically 7 or 19) from the structure. PBGFs are characterized by well-defined low loss transmission windows, the spectral position of which is determined by the structural parameters of the photonic crystal cladding, primarily the hole to hole spacing and the relative hole size. Anti-resonant (AR) fibres have generally a simpler cladding structure, which is not required to be periodic. The transmission properties are determined by the thickness and shape of the thin glass struts in the cladding and of the core surround. Optical guidance in this case is achieved due to frustrated coupling between core and cladding modes and by suitably designing the thickness of the core boundary. Differently from PBGFs, AR fibres can operate over very broad wavelength intervals with losses that are now approaching the $0.01 \mathrm{~dB} / \mathrm{m}$ level. Hollow core microstructured fibres are characterized by high damage thresholds, extremely low nonlinearity and extended transmission at infrared wavelengths, and are thus of interest for applications such as low latency (speed-of-light-in-air) communications, gas sensing and nonlinear spectroscopy, and high-peak power pulsed laser delivery. In this work we report the improvements in the fabrication of these challenging fiber waveguides, in particular the fabrication of low-loss, wide bandwidth (>100nm) PBGFs operating at $2 \mu \mathrm{m}$, and anti-resonant "hexagram" fibres with broadband transmission for the delivery of extremely high peak optical powers.

\section{Development of low loss, wide bandwidth Photonic Bandgap Fibers at $\mathbf{2} \boldsymbol{\mu m}$}

The transmission properties of PBGFs differ quite significantly from those of conventional fibres. The fiber attenuation, which can be as low as $\sim 1 \mathrm{~dB} / \mathrm{km}$ at $1550 \mathrm{~nm}$, is determined by scattering at the air/glass core interface which arises due to roughness induced during fabrication by surface capillary waves [2]. Rayleigh scattering, which is the limiting loss mechanism in conventional fibers, is negligibly small in gases and infrared absorption only becomes significant at longer wavelengths, by virtue of the reduced overlap (typically $\leq 0.1 \%$ ) between the guided mode and glass in these structures. Laser beam delivery applications typically only require short device lengths (a few meters to a few tens of meters) and thus are less demanding in terms of transmission loss. Comparatively more important are issues concerning the modal quality of the output beam. In this respect, it is important to recognize that the most common types of PBGFs are able to support several air guided modes, although they often can be operated in a quasi-single-mode regime over short lengths by optimizing the input coupling to the fundamental mode. PBGFs also support interface or surface modes (SMs) which have strong overlap with the glass structure and thus show significantly higher loss. Where they resonantly couple to air guided modes, SMs appear as additional loss peaks within the transmission window. SMs are undesirable as they reduce the useable transmission bandwidth and introduce coupling between the fundamental and higher order air guided modes. Coupling to SMs also radically changes the dispersion properties of the PBGF, which is undesirable for delivery of highpeak power pulsed beams.

The number, position and strength of the loss peaks due to SMs are a function of the structure of the core ring boundary, and in particular its thickness, radii and ellipticity. Control and even elimination of SMs is possible in PBGFs based on smaller core defects (i.e. 3-cell [3] and 7-cell [4]), but becomes substantially more challenging in structures with large core sizes (19-cell) due to difficulties in achieving an optimal core structure [5]. On the other hand, 19c PBGFs are interesting for power delivery applications due to their higher damage threshold as compared to their smaller core counterparts [6]. Previous studies have concentrated on the loss reduction aspect and achieved loss as low as $1.7 \mathrm{~dB} / \mathrm{km}$; however, the particular choice of the design of a 'thick' core boundary meant that a substantial compromise in terms of SMs and transmission bandwidth was unavoidable. We have therefore investigated different design regimes, characterised by a relatively "thin" core boundary, i.e. about one half the thickness of the cladding struts [3, 4]. We concentrated on the wavelength region around $2 \mu \mathrm{m}$. This wavelength region is becoming increasingly important due to impressive progress of high-power Tm lasers and is also predicted by theory to yield the lowest transmission low in a PBGF.

We successfully fabricated 19-cell PBGFs exhibiting unprecedented combination of record low loss and wide transmission bandwidth centered between 1.9 and $2.1 \mu \mathrm{m}$. Figure 4 shows a fiber with a minimum loss of $6.8 \mathrm{~dB} / \mathrm{km}$ 
(measured over a $230 \mathrm{~m}$ cutback) and a $3-\mathrm{dB}$ bandwidth of approximately $160 \mathrm{~nm}$. The PBGF was fabricated using a twostep stack and draw technique [3]. The central core was obtained without the use of a central element in order to avoid increasing the wall thickness of the core, thus minimizing the guidance of surface modes in the final fiber.
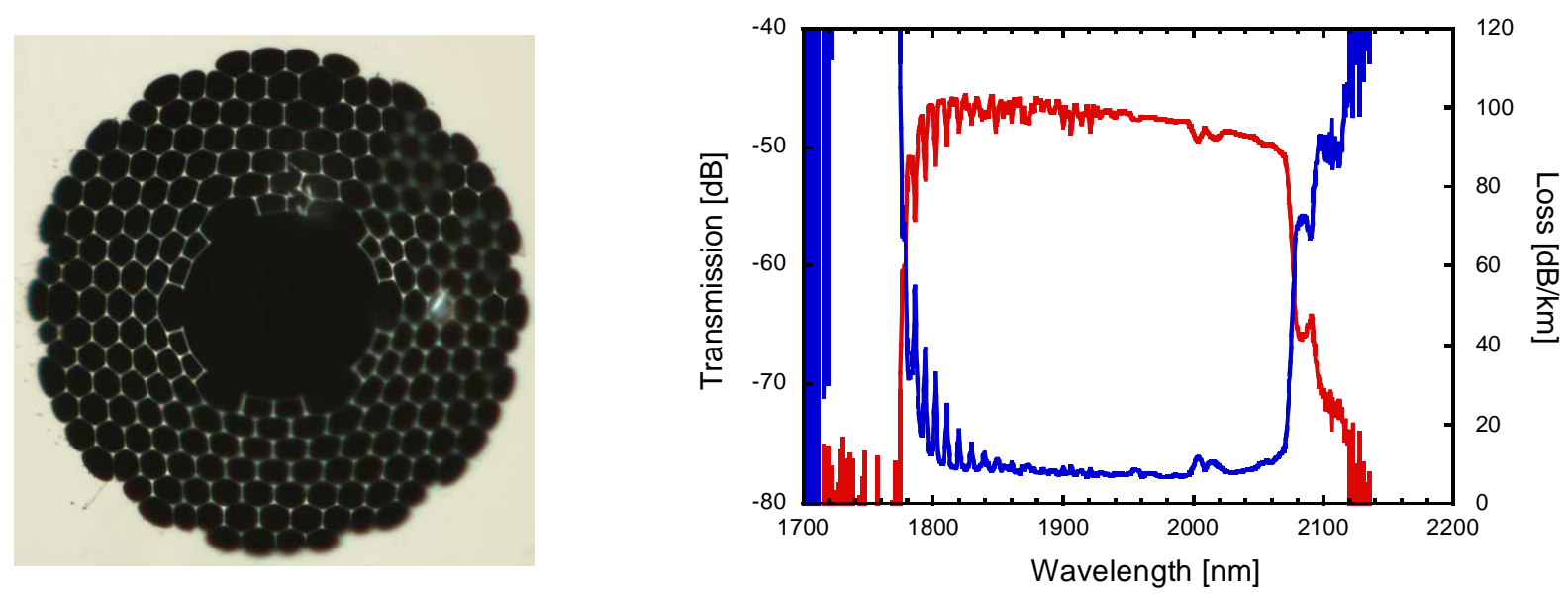

Figure 4: Transmission loss of a hollow core PBGF designed for operation at $2 \mu \mathrm{m}$ with minimum loss of $6.8 \mathrm{~dB} / \mathrm{km}$ at $\sim 1980 \mathrm{~nm}$ and $3 \mathrm{~dB}$ bandwidth of approximately 160nm (limited by gas absorption).

It can be seen that the central section of the transmission windows are completely surface mode-free, however bands of narrow absorption lines from gaseous species contained in the PBGF core can clearly be identified. The band at 1.8$1.95 \mu \mathrm{m}$ is due to water vapor, while two weaker bands at $1.96 \mu \mathrm{m}$ and $2.01 \mu \mathrm{m}$ originate from carbon dioxide. Further absorption lines observed between $1.7-1.85 \mu \mathrm{m}$ have been attributed to hydrogen chloride [7]. It is to be noted that no particular precaution was taken during the various fabrication steps in order to minimize the presence of these species inside the fiber, nor to avoid subsequent ingress of atmospheric gases into the fibres while they were stored or characterized. It is therefore plausible that the concentration of atmospheric absorbing gas species (such as $\mathrm{H}_{2} \mathrm{O}$ and $\mathrm{CO}_{2}$ ) can be significantly reduced through improvements of the fabrication procedure. From HITRAN data, the absorption strength of the strongest lines of $\mathrm{H}_{2} \mathrm{O}$ at $1.87 \mu \mathrm{m}$ is $\sim 1 \mathrm{~dB} / \mathrm{km} / \mathrm{ppmV}$; the peak absorption of $\mathrm{HCl}$ is $0.6 \mathrm{~dB} / \mathrm{km} / \mathrm{ppmV}$ at $1.75 \mu \mathrm{m}$; while the peak absorption of $\mathrm{CO}_{2}$ is approximately $0.06 \mathrm{~dB} / \mathrm{km} / \mathrm{ppmV}$ at $2.01 \mu \mathrm{m}$. The presence of these gas species in sufficient proportion has therefore the potential to significantly increase the transmission loss and decrease the usable bandwidth of PBGFs designed for $2 \mu \mathrm{m}$ operation and must therefore be carefully avoided.

\section{'Hexagram' Antiresonant Fibers}

Antiresonant microstructured fibres [8] confine light in a hollow core over broad wavelength intervals in which light is antiresonant with the thin glass core surround. At wavelengths where light is resonant with the core surround high confinement loss occurs. Many structures are possible, but our interest lies in obtaining good performance from the simplest structures which can be readily fabricated using the stack and draw technique. By stacking 6 capillaries in an outer jacket tube, fiber with a hollow core and hexagonal glass core surround supported by thin struts may be formed. By controlling pressure in the spaces defined by the capillaries, the core surround may be supported by either radial struts or result in a hexagram-type structure (Figure 5). In order to investigate the relative merits of these two designs in terms of confinement loss we performed numerical simulations on both structures and on a reference structure having an unsupported circular core surround. Our calculations and comparison with the reference structure showed that segmentation of the core surround, the geometry of the support struts and the separation between core surround and the outer glass jacket all influenced confinement loss [9]. In particular the hexagram arrangement of support struts gave the 
lowest loss at most wavelengths, although with some compromise in term of stronger oscillations in the spectral transmission.
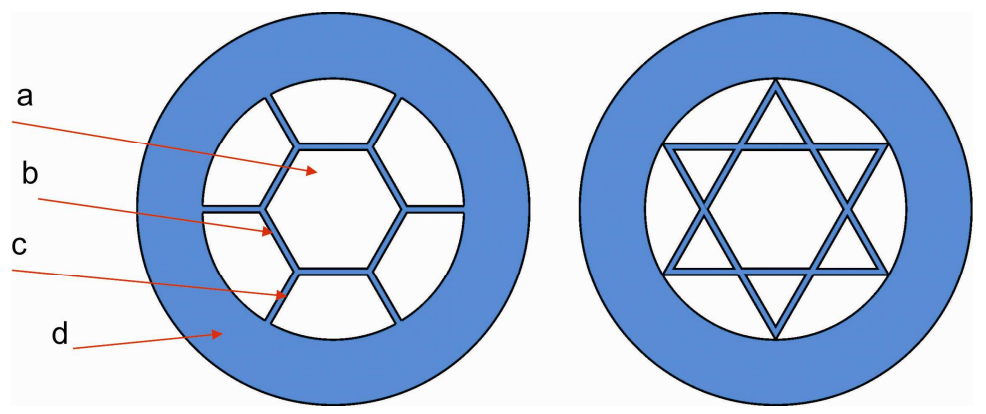

Figure 5. Essential features of simple antiresonant fibres. A large hollow core (a) is surrounded by a thin glass ring (b) supported by thin glass struts (c) and enclosed by a glass jacket (d).
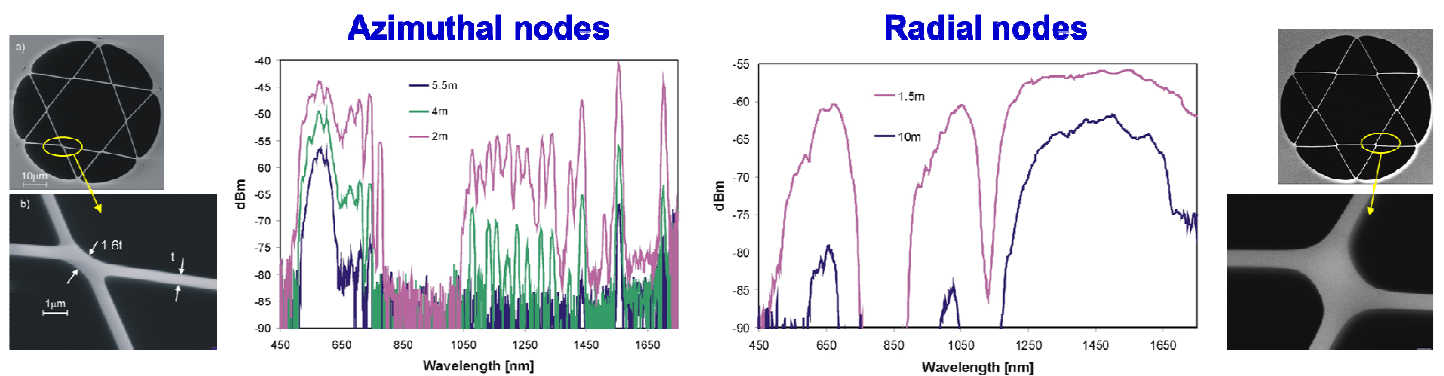

Figure 6. Electron micrographs and transmission spectra of hexagram fibres. Nodes may be aligned azimuthally or radially. Both fibre shown have resonances close to $450 \mathrm{~nm}$ and $850 \mathrm{~nm}$ that are inherent to the guidance mechanism. The transmission spectrum of the radially aligned node fiber has far fewer unwanted additional resonances.

We carried out a systematic investigation of the origin of such oscillations. Due to surface tension, nodes of thickened glass at the intersections of the support struts form during the fiber draw. Their effect is to introduce many additional high loss resonances (confirmed by numerical simulation) and prevent attainment of the losses predicted by calculations for an idealized unsupported circular core. Through careful control of differential pressure during fiber draw these nodes may be aligned along the surround (azimuthal nodes) or perpendicular to it (radial nodes). We find that the transmission spectrum of fiber with radially aligned nodes has resonant features due to the nodes greatly reduced both in number and in strength (Figure 6). By contrast, fibres with azimuthal nodes have highly structured transmission spectra and only very narrow ( a few tens of nm wide) low loss windows. To date our lowest loss in a fiber with azimuthal modes was $0.4 \mathrm{~dB} / \mathrm{m}$ at a wavelength of $2.17 \mu \mathrm{m}$ in a $77 \mu \mathrm{m}$ diameter core. Due to a technical problem we have yet to draw a sufficiently long of radially aligned node fiber with consistent dimensions to allow for loss measurements by cutback. However we have demonstrated good bend loss performance using a $1.5 \mathrm{~m}$ length of fiber having a core width of $45 \mu \mathrm{m}$. At wavelengths between $1.2 \mu \mathrm{m}$ and $1.75 \mu \mathrm{m}$ (long wavelength limited by our optical spectrum analyzer) the loss induced by one turn was less than $0.5 \mathrm{~dB}$ for a bend radius of $4 \mathrm{~cm}$. Figure 7 shows the gaussian-like mode at the fiber end face obtained using controlled excitation and a Si-CCD camera with side illumination to show the fiber structure. From this we obtained an estimated mode field diameter of $39 \mu \mathrm{m}$.

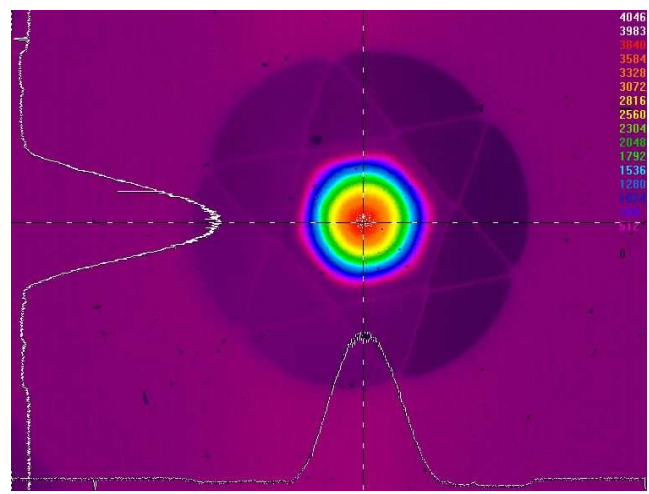

Figure 7. Near field image of mode in a hexagram fiber with radially aligned nodes and a core width of $45 \mu \mathrm{m}$. Side illumination is used to show the fiber structure. 


\section{CHALCOGENIDE GLASSES AND FIBRES}

The exploitation of chalcogenide materials has evolved from a simple infrared transmitting bulk glass into a multifunctional optoelectronic material for current and future use. Amongst their many applications, chalcogenide glasses provide a powerful material base for mid-IR photonic and electro-optical applications. These currently include optical fibre, active plasmonic waveguides, optically and electrically switchable thin films, metamaterials and solid state lasers, amongst others. Being in the core of rewritable DVDs and CDs, this robust technology is finding new applications provided by the combination of wide transmission band, high optical nonlinearity and the unique phase change switching functionality of advanced chalcogenide glasses. We describe our work with gallium and germanium sulphide based glasses and their potential for improved low loss IR fibre for the 3-5 micron transmission window.

\section{Purification and Synthesis}

For both the practical application and scientific study of chalcogenide glasses, glass purity is of utmost importance. The reasons are both scientific and practical. High purity glass ensures repeatability of experimental work, particularly when dealing with active properties of the glasses. Varying levels of trace impurities, even at levels of a few parts per million can alter the spectroscopic behaviour of a glass. Impurities in the raw materials and hence in the resulting glass, contribute to the loss of power through any optical component, whether it is in the form of a long glass fiber, an infrared window or the whispering gallery mode of a microsphere. These impurities contribute to the optical loss through absorption and scattering as well as serving as nucleation sites for crystallization, which even in applications where glass to crystalline phase changes are desired, must still be reproducible and controlled. Although high purity raw elements are now commercially available, with $99.9999 \%$ purity routine for many metals, even this level of purity is often not sufficient, particularly for optical fiber applications [10]. This is more of a concern with commercially available chalcogenide compounds such as germanium sulphide, gallium sulphide or arsenic sulphide. Although these may have been synthesized from high purity elements, the conversion process itself can readily introduce oxide, water or organic impurities. It is not unheard of to find for example, commercial gallium sulphide contaminated with $45 \%$ or more gallium oxide through incomplete reaction of the precursors during production.

\section{Chemical Vapour Deposition}

Chemical vapor deposition (CVD) is a chemical process used to produce high-purity, solid materials from liquid and gaseous precursors. Reaction, for example, between germanium chloride $\left(\mathrm{GeCl}_{4}\right)$ and hydrogen sulphide $\left(\mathrm{H}_{2} \mathrm{~S}\right)$ was reported by Huang et al, using high purity germanium chloride $(99.9999 \%)$ reacted with hydrogen sulphide to form germanium sulphide where the ratio of Ge to $\mathrm{S}$ could be controlled through the temperature of the reaction chamber [11]. CVD can be a complicated deposition process since there are several factors occurring simultaneously and nonuniformly whilst in the gas phase. These factors include: forced convection (flow due to pressure gradients), free convection (flow due to buoyancy of hot gas), homogeneous reactions and diffusion.

Nonetheless, it is clear that the levels of transition metal impurities are much lower than those observed for chalcogenide glasses melted using conventional techniques. Using a similar technique, Katsuyama [12] synthesized Ge-Se particles by reaction of halide precursors with $\mathrm{H}_{2}$. This reaction took place directly within a silica tube which was then sealed off and used for glass melting and subsequent fibre drawing.

\section{Glass Melting}

The conventional method for producing chalcogenide glasses is through the use of sealed ampoule melting. In this technique the required glass precursor materials are sealed under vacuum in a silica ampoule, melted, and then quenched to form a glass within the ampoule [13]. The requirement for the sealed atmosphere is dictated by the volatile nature of many of the precursors which if melted in open atmosphere can result in large compositional changes or complete removal of components with low vapour pressures. This process also has the effect of trapping any impurities in the precursors within the glass as thus the precursor purity limits the ultimate quality of the glass that is produced. In addition, impurities can be transferred to the glass from the ampoule walls. Chalcogenides melted from compounds such as gallium and lanthanum sulphide have the advantage that they do not require a sealed system therefore glass melting can take place in a flowing stream of high purity or reactive gas. 


\section{Optical Fibre}

Chalcogenide optical fibers have for several decades been technologically important; they can transmit in the 2-12 $\mu \mathrm{m}$ wavelength region, beyond the transmission window of conventional optical fiber and can therefore enable numerous infrared applications. Optical fibers drawn from chalcogenide based glasses were first investigated in the 1970's when fibers from glasses based on arsenic or germanium selenides and tellurites rapidly found application for transmission of carbon dioxide $\left(\mathrm{CO}_{2}\right)$ laser wavelengths around 10 microns.

There are two basic methods of drawing fiber: from a pre-prepared preform or from a melt. Fiber drawing from chalcogenide glasses has been extensively reviewed and several excellent publications describe the various fabrication techniques in detail [10,14-17]. For preform drawing, a glass rod which consists of a central core rod and an outer cladding tube is used. Variations of traditional melt casting techniques such as built-in-casting and rotational casting [15] have been adapted to process chalcogenide glass into the required structure. In addition, alternative preform fabrication processes have been developed such as the rod in tube process. In this process, the core glass rods are obtained by direct melting and quenching in the quartz ampoules and can therefore be made to almost any diameter and length. These ingots can then be ground and polished down to any desired diameter. The cladding glass tubes are made by spinning the molten cladding glass in the quartz ampoule while cooling the assembly to the glass transition temperature and then annealing. Most recently, extrusion techniques have been developed to provide the desired core/clad structure [18].

The preforms are subsequently drawn into fibres on a fiber drawing tower in the viscosity range of 104-106 Poise. The preform feed rate into the furnace, the furnace temperature and the capstan/winding drum speed are used to control the fiber diameter. The preform can be initially coated with a heat shrinkable Teflon tube and drawn into fiber or an UVcurable acrylate or thermoset coating can be applied in-line. Figure 8 shows the internal structure of a multimode Ga-La$\mathrm{S}$ fiber drawn from a perform fabricated by extrusion. The core diameter of approximately 15 microns is surrounded by an inner cladding about 5 microns in thickness, a second inner cladding which makes up the bulk of the fiber and a final out 5 micron thick outer cladding. This multilayer structure is a consequence of the extrusion process. Here the

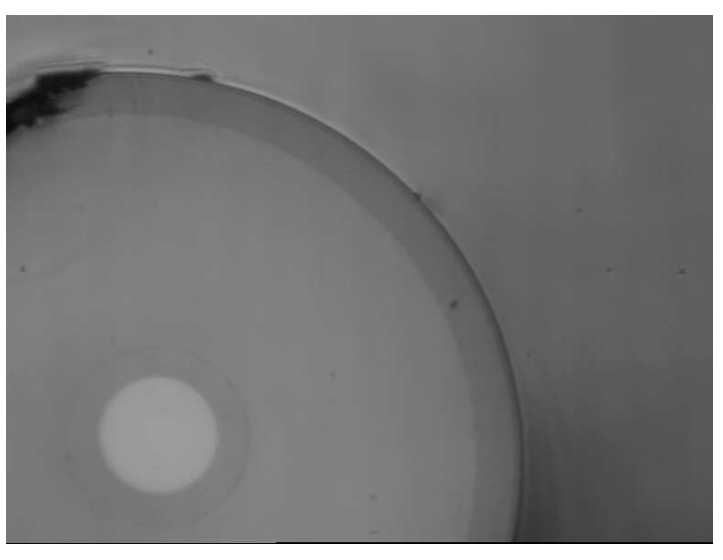

In the crucible drawing process, the core and cladding glasses are re-melted in concentric quartz crucibles which have small holes in their bases [19]. Once the core melt has reached a viscosity of $10^{3}-$ $10^{5}$ Poise, slightly lower than with preform drawing, it flows through the hole and into the cladding glass melt where upon both core and cladding glasses flow out the second hole in the bottom of the crucible. Under optimized processing conditions, the ensuing fibre possesses a defect-free and concentric core/clad structure. The processing is performed under an inert atmosphere of argon. The fibre diameter is controlled by independent pressure control above the core and cladding glass melts, respectively, as well as the furnace temperature and draw speed. Again, an outer polymer coating, such as an UV curable acrylate or thermoset polymer, is applied in-line. The double crucible process can be used to make multimode and single mode fibres by appropriate control of the

Figure 8. Cross section of a gallium lanthanum sulphide optical fibre formed from an extruded preform [18].

\section{Applications}

The diversity of both the properties of chalcogenide glasses and the geometries into which they can be formed have ensured current and future interest in the application of these emerging optoelectronic family of materials. Bulk optics and optical fibers were the initial drivers for chalcogenide glass research and development. Interest continues strongly in both these areas but today many promising new applications, based for example on chalcogenide films, from nanometer to millimeter thicknesses are being studied. Other applications are exploiting microspheres, nanoparticles and nanowires formed from these incredibly versatile glasses. 


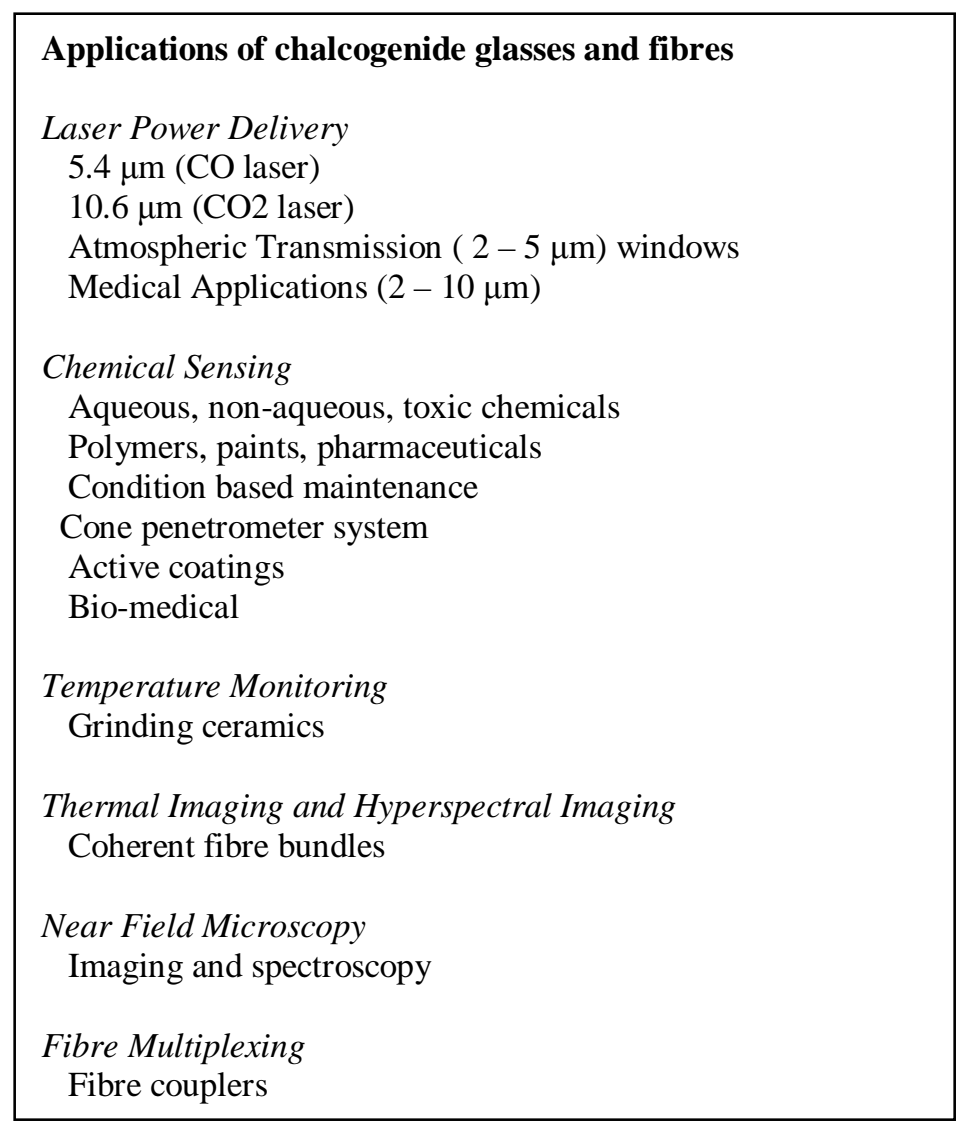

\section{CONCLUSIONS}

We have described some of the recent developments in optical fibre technology made possible by a variety of new design concepts in conjunction with advances in fibre fabrication techniques. Nanomechanical optical fibres have been realized where optical switching inside the fibre is achieved by imparting nanometer-scale motion to the core, holding out promising prospects for integrated fibre multifunctional devices. Conversely, such fibres could lead to the development of high sensitivity optical sensors, capable of detecting nanometer movement along the fibre. Microstuctured fibres comprising a hollow air core with reasonable optical transmission performance over select wavelength bands have been developed, with prospects for extended IR transmission in silica-based fibres, high power (high damage threshold) laser delivery, and even optical fibre gas lasers. Developments in chalcogenide glasses and fibres are increasingly driven by a range of applications beyond simple IR transmission, including potential plasmonic and metamaterials applications.

\section{ACKNOWLEDGEMENTS}

The authors gratefully acknowledge support from the Engineering and Physical Sciences Research Council via the EPSRC Centre for Innovative Manufacturing in Photonics (grant reference EP/H02607X/1). 


\section{REFERENCES}

[1] Z. G. Lian, et al., "Optical fiber with dual cores suspended in air", European Conference on Optical Communication (ECOC) Geneva, Switzerland 18-22 September (2011).

[2] P. J. Roberts, et al., "Ultimate low loss of hollow-core photonic crystal fibres," Opt. Express, 13, pp. 236-244 (2005).

[3] M. N. Petrovich et al., "Robustly single mode hollow core photonic bandgap fiber," Opt Express 16, 43374346 (2008).

[4] R. Amezcua-Correa, et al., "Control of surface modes in low loss hollow-core photonic bandgap fibers," Opt. Express, vol. 16, pp. 1142-1149 (2008).

[5] R. Amezcua-Correa, et al., "Design of 7 and 19 cells core air-guiding photonic crystal fibers for low-loss, wide bandwidth and dispersion controlled operation," Optics Express, vol. 15, pp. 17577-17586 (2007).

[6] J.D. Shephard, et al., "Improved hollow-core photonic crystal fiber design for delivery of nanosecond pulses in laser micromachining applications," Appl. Opt. 44, 4582-4588 (2005).

[7] J. K. Lyngso, et al., "7-cell core hollow-core photonic crystal fibers with low loss in the spectral region around $2 \mu \mathrm{m}, "$ Opt. Express, vol. 17, pp. 23468-23473 (2009).

[8] N. M. Litchinitser, A. K. Abeeluck, C. Headley, and B. J. Eggleton, "Antiresonant reflecting photonic crystal optical waveguides," Opt. Lett. 27(18),1592-1594 (2002).

[9] F. Poletti, J.R. Hayes, D.J. Richardson "Optimising the performances of hollow antiresonant fibres", European Conference on Optical Communication (ECOC) Geneva, Switzerland 18-22 September (2011)

[10] J.S. Sanghera and I. D. Aggarwal, Editors, "Infrared Fiber Optics", CRC Press, LLC, Florida (1998).

[11]C.C.Huang, D.W.Hewak and J.V.Badding, "Deposition and characterization of germanium sulphide glass planar waveguides", Optics Express, 12:2501-2505, 2004.

[12] T. Katsuyama, S. Sathoh and H. Matsumura, "Fabrication of high-purity chalcogenide glasses by chemical vapour deposition", J. Appl. Phys. 59:1446, 1986.

[13] J. S. Sanghera, L. B. Shaw, L. E. Busse, V. Q. Nguyen, P. C. Pureza, B. C. Cole, B. B. Harbison, I. D. Aggarwal, R. Mossadegh, F. Kung, D. Talley, D. Roselle, and R. Miklos, "Development and infrared applications of chalcogenide glass optical fibers," Fiber Integr. Opt., 19:251-274, 2000.

[14] D.J. Brady, PhD Thesis, University of Southampton, Southampton, UK, 1999.

[15] P.W. France, editor, "Fluoride Glass Optical Fibers", Blackie, London, 1989.

[16] B.J. Ainslie, K.J. Beales, C.R. Day and J.D. Rush, "The design and fabrication of monomode optical fiber", IEEE J. Quant. Electon., 18:514 1982.

[17] T. Katsuyama and H. Matsumura, "Infrared Optical Fibers", Adam Hilger, IOP Publishing Ltd., 1989.

[18] M.N. Petrovich, PhD Thesis, Universisty of Southampton, Southampton, UK, 2003.

[19] J. S. Sanghera, L. B. Shaw, L. E. Busse, V. Q. Nguyen, P. C. Pureza, B. C. Cole, B. B. Harbison, I. D. Aggarwal, R. Mossadegh, F. Kung, D. Talley, D. Roselle, and R. Miklos, "Development and infrared applications of chalcogenide glass optical fibers," Fiber Integr. Opt., 19:251-274, 2000. 